\title{
A technique for constructing diverting loop ileostomy to prevent outlet obstruction after rectal resection and total colectomy: a retrospective single-center study
}

\author{
Yusuke Takehara ${ }^{1} \cdot$ Mihoko Nakagawa $^{1} \cdot$ Hiroaki Kobayashi ${ }^{1} \cdot K$ Kensuke Kakisako ${ }^{1} \cdot$ Yojiro Takano $^{1}$ - Junichi Seki ${ }^{1}$. \\ Shoji Shimada ${ }^{1} \cdot$ Kenta Nakahara $^{1} \cdot$ Shumpei Mukai ${ }^{1} \cdot$ Yuta Enami $^{1} \cdot$ Naruhiko Sawada $^{1} \cdot$ Fumio Ishida $^{1}$. \\ Shin-ei Kudo ${ }^{1}$
}

Received: 6 May 2021 / Accepted: 8 July 2021 / Published online: 24 October 2021

(c) The Author(s) 2021

\begin{abstract}
Purpose Preventing outlet obstruction associated with a diverting stoma is important. Previously, we constructed a diverting loop ileostomy with the proximal limb of the small intestine on the caudal side, namely the oral inferior (OI) method. However, to address the issue of twisting and stenosis of the small intestine, we recently constructed a diverting loop ileostomy with the proximal limb on the cranial side, namely the oral superior (OS) method. We compared the incidence of outlet obstruction between the two methods.

Methods The subjects of this retrospective study were 133 patients who underwent colorectal resection or total colectomy, with D2 or more lymph node dissection and diverting loop ileostomy construction, between April, 2001 and December, 2018, at our hospital. The OI method was performed in 54 patients and the OS method was performed in 79 patients.

Results In the OS group, a history of laparotomy, neoadjuvant therapy, clinical stage III, and the use of anti-adhesion materials were more common, whereas blood loss and the incidence of outlet obstruction were significantly lower. Multivariate analysis identified only OS placement as a significant factor for reducing the incidence of outlet obstruction.

Conclusion When constructing a diverting loop ileostomy, placing the proximal limb on the cranial side is important.
\end{abstract}

Keywords Outlet obstruction · Diverting loop ileostomy $\cdot$ Oral superior

\section{Introduction}

Recent remarkable advances in preoperative chemoradiation therapy and surgical techniques for lower rectal cancer and the widespread use of this treatment combination have led to an increase in the number of cases of diverting stoma construction to reduce the risk of postoperative leakage [1,2]. However, no consensus exists regarding whether ileostomy or colostomy is better [3-6]. In Japan, ileostomy is often selected because it is easy to construct and close [7]. Stomarelated complications include skin disorders, stoma necrosis, stoma prolapse, high-output, parastoma hernia, and bowel

Yusuke Takehara

yuutake47@yahoo.co.jp

1 Digestive Disease Center, Showa University Northern Yokohama Hospital, 35-1 Chigasaki-chuo, Tsuzuki-ku, Yokohama, Kanagawa Prefecture 224-8503, Japan obstruction [8-10]. In particular, bowel obstruction, called outlet obstruction (OO), tends to occur with loop ileostomy. Differentiating between $\mathrm{OO}$ and bowel obstruction/ileus is difficult because both cause bloating and vomiting, and a diagnosis of bowel obstruction may include OO. However, ileus and $\mathrm{OO}$ are separate pathological entities with different causes because ileus may present as diffuse dilation down to the stoma site, whereas $\mathrm{OO}$ involves an obstruction at the stoma site. $\mathrm{OO}$ may interfere with early meal initiation and necessitate early stoma closure. OO was initially reported as ileostomy with ileus after surgery for ulcerative colitis (UC) or familial adenomatous polyposis (FAP) [11-13], but the number of reports of $\mathrm{OO}$ has increased in recent years [11-17]. Several reports on the prevention of OO indicate that a fascial incision should be made vertically rather than by a cross incision to reduce adhesion and twisting of the mesentery, and to create a stoma tunnel with a sufficient margin [8-10, 18, 19]. Furthermore, laparoscopic surgery has been reported as a risk factor for $\mathrm{OO}[20]$ and has not yet 
been considered for preventing OO [8-10, 12, 19, 20]. However, unlike other complications associated with a stoma, the incidence of $\mathrm{OO}$ is expected to be reduced by revising the procedure during stoma construction. Therefore, prevention strategies are important when constructing a diverting loop ileostomy (DLI) $[9,12,19]$. Several reports indicate that rotating the proximal limb of the small intestine to the caudal side is better when constructing loop ileostomy; however, it is unclear if rotation of the small intestine can prevent $\mathrm{OO}$ $[18,19,21-23]$. In the past, when constructing a DLI in our department, the oral side was the caudal side (oral inferior; OI). However, now we construct it so that the oral side is the cranial side (oral superior; OS) because of recent findings of twist and stenosis of the small intestinal limb. We conducted this retrospective study to examine the incidence of $\mathrm{OO}$ after the OI method versus the OS method.

\section{Methods}

Between April, 2001 and December, 2018, 165 patients underwent rectal resection or total colectomy with lymph node dissection of $\mathrm{D} 2$ or more, with intraoperatively constructed DLI in the right upper or lower abdomen performed laparoscopically. After excluding patients with bowel obstruction caused by colorectal cancer before surgery; those who underwent emergency surgery for reasons such as peritonitis; those with distant cancer metastasis, multiple cancers, or multiple primary cancer; and those with postoperative complications of Clavien-Dindo IIIb or more, 133 patients who had undergone stoma closure were the subjects of this analysis [24] (Fig. 1). There were 54 patients in the OI group and 79 patients in the OS group. The procedure for constructing a DLI in our department can be summarized as follows:

The stoma site was marked before surgery, based on the principles of the Cleveland Clinic (Cleveland, $\mathrm{OH}$, USA). After making a 3- to 4-cm longitudinal skin incision with the apex marked, the rectus abdominis sheath was also incised vertically to the same length. The rectus abdominis muscle was split bluntly to form a stoma tunnel that allowed two lateral fingers to pass sufficiently. After pulling out the small intestine on the oral side, approximately $30-40 \mathrm{~cm}$ from the terminal ileum, based on the Brooke method, a loop ileostomy was constructed with approximately 8-10 strands of absorbable suture material on needles, to a height of approximately $5 \mathrm{~mm}$ on the anal limb and approximately $30 \mathrm{~mm}$ on the proximal limb. Laparoscopically, the ileum was followed from the terminal ileum to confirm that the small intestine on the oral side, approximately $30-40 \mathrm{~cm}$ from the terminal ileum, could be elevated to the marked stoma site. To prevent pneumoperitoneum, after returning the patient to the supine position, we constructed the loop ileostomy

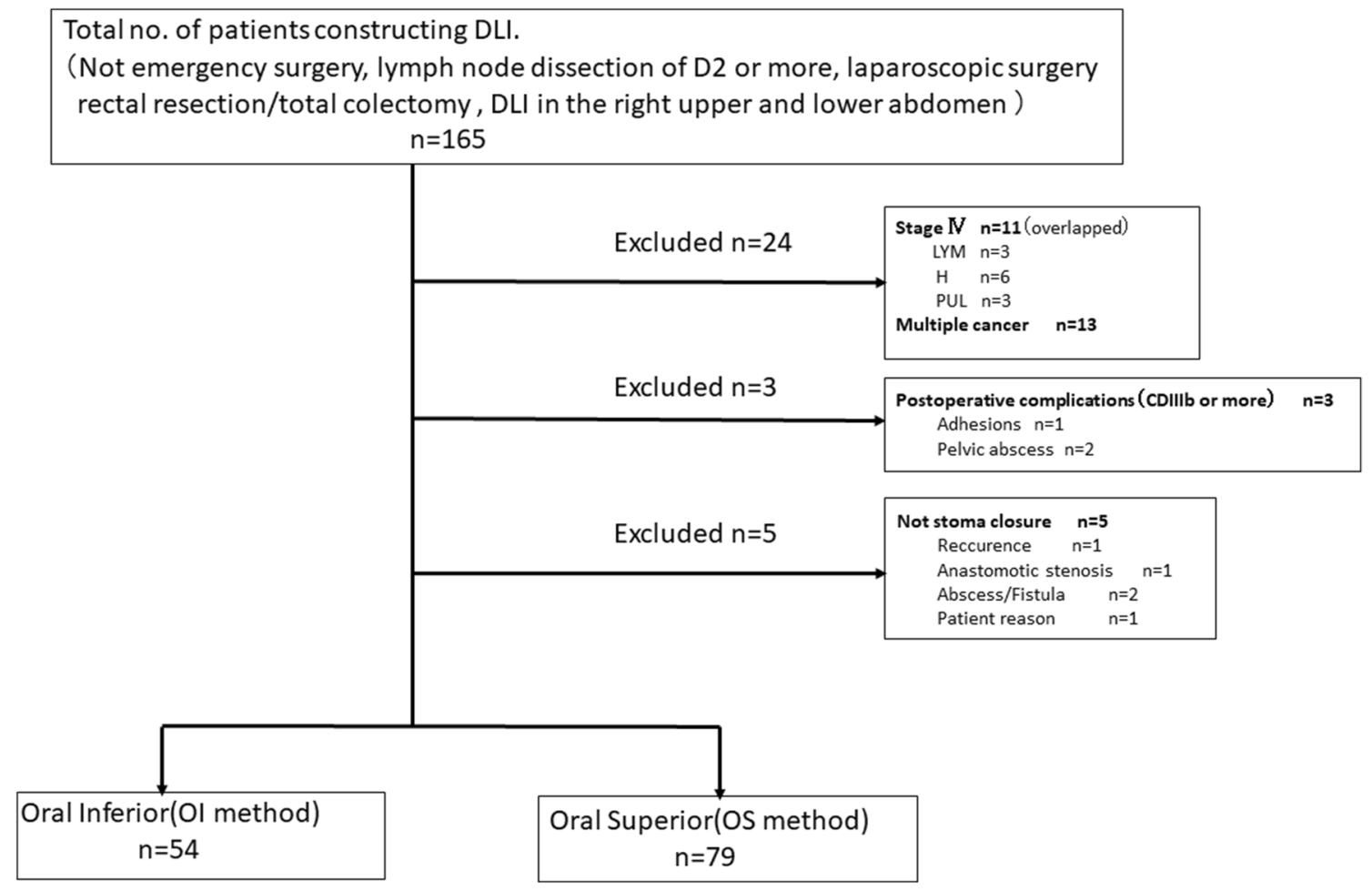

Fig. 1 The CONSORT diagram for this study. CONSORT Consolidated Standards of Reporting Trials 
using the aforementioned method, confirming it directly through a small laparotomy wound. At the conclusion of open and laparoscopic surgery, the main abdominal wound was closed and dressings were applied to exclude it from contamination when the loop ileostomy was opened.

We compared the following factors between the two groups: sex; age; history of laparotomy; body mass index; primary disease (colorectal cancer: neuroendocrine tumor/ UC/FAP); neoadjuvant therapy; clinical stage (using the criteria of UICC TNM Classification of Malignant Tumours, 8th edition [25]; stage 0-II:III); performance status of $0-1: 2-4$; surgical procedure, such as rectal resection or total colectomy such as ileal pouch anal anastomosis (IPAA); whether anti-adhesion material such as Seprafilm [Baxter International Inc., Deerfield, IL, USA]) was used; operation time; blood loss; postoperative length of stay in hospital; period until stoma closure; OO; stoma-related complications, excluding bowel obstruction/ileus and $\mathrm{OO}$; the degree of intra-abdominal adhesion; rectus abdominis muscle thickness (horizontal and vertical); length of the straight line connecting both ends of the rectus abdominis muscle; the position where the stoma limbs penetrated the rectus abdominis muscle (center-inside or outer); and the angle between the stoma limbs and the rectus abdominis muscle.

The features of $\mathrm{OO}$ are as follows: (1) symptoms of intestinal obstruction such as bloating and vomiting; (2) relief of the bowel obstruction by inserting a decompression catheter trans-stomally; and (3) computed tomography (CT) image of a caliber change in the abdominal wall-penetrating part of the stoma but no other obstruction mechanism. Criteria 1 and 2 or 1 and 3 were satisfied during the period from construction of the DLI to stoma closure.

The horizontal thickness $(\mathrm{mm})$ of the rectus abdominis muscle was measured by selecting an umbilical level slice in the preoperative $\mathrm{CT}$ examination and drawing a straight line perpendicular to the horizontal axis at the thickest part of the right rectus abdominis muscle in the image [26] (Fig. 2a). The vertical thickness $(\mathrm{mm})$ of the rectus abdominis muscle was measured by selecting an umbilical level slice in the preoperative CT examination and drawing a straight line perpendicular to the straight line connecting both ends of the rectus abdominis muscle [27] (Fig. 2b). The thickness was classified as $<10 \mathrm{~mm}$ or $\geq 10 \mathrm{~mm}$. The length of the straight line connecting both ends of the rectus abdominis muscle was measured by selecting an umbilical level slice in the preoperative CT examination.

\section{Rectus abdominis muscle thickness (horizontal) Rectus abdominis muscle thickness (vertical)}

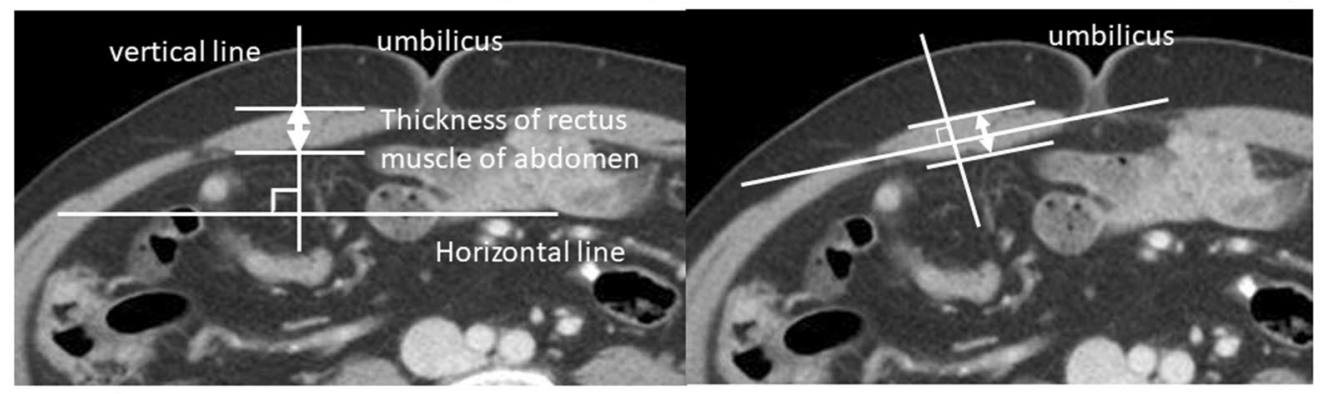

$\mathbf{a}$

b

The position of the stoma limbs

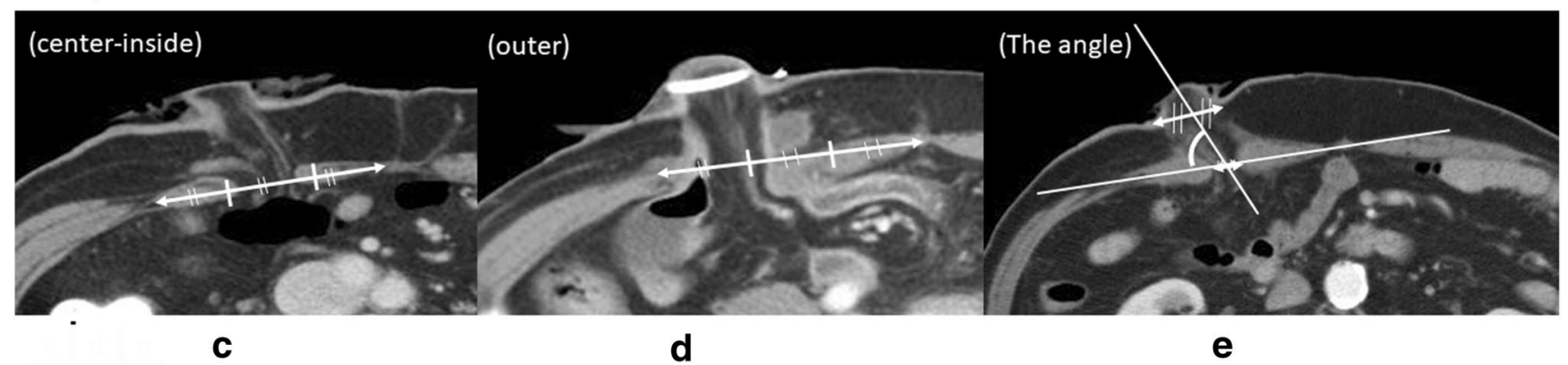

Fig. 2 a A straight line is drawn perpendicular to the horizontal axis at the thickest part of the right rectus abdominis muscle. b A straight line is drawn perpendicular to the straight line connecting both ends of the rectus abdominis muscle. c The stoma limbs are classified as penetrating the center-inside. d The stoma limbs are classified as pen- etrating the outside. e The angle formed by the straight line connecting both ends of the rectus abdominis muscle and the long axis of the stoma limbs is measured on a CT scan image and on the slice in which the stoma limbs penetrate the rectus abdominis 
The position where the stoma limbs penetrate the rectus abdominis muscle was identified by $\mathrm{CT}$ examination before stoma closure in the 64 patients who underwent CT examination before stoma closure. On dividing the straight line connecting both ends of the rectus abdominis into three equal parts, the stoma limbs were classified as penetrating the center-inside (Fig. 2c) or the outside [27] (Fig. 2d). The angle between the stoma limbs and the rectus abdominis was calculated, based on CT scan images before stoma closure. The slice in which the stoma limbs penetrated the rectus abdominis was selected. The angle formed by the straight line connecting both ends of the rectus abdominis muscle and the long axis of the stoma limbs was measured [27] (Fig. 2e). The long axis of the stoma limbs was defined as the line connecting the centers of both short axes, where the stoma limbs penetrate the rectus abdominis muscle and the epidermal level [27] (Fig. 2e).

We evaluated the incidence, extent, and type of adhesions in the abdominal cavity of adhesions during the second laparotomy for stoma closure. A circular laparotomy wound was made around the stoma, which revealed adhesions in the abdominal cavity. Existing adhesions around the midline incision and peristomal area were evaluated as "none" for no adhesions; "mild," for adhesions covering up to $25 \%$ of the total area and length with a filmy thickness and avascularity; "moderate," for adhesions covering $26 \%-50 \%$ of the total area and length, moderate thickness, and limited vascularity; and "severe," for adhesions covering $>51 \%$ of the total area and length with dense thickness and vascularization. We examined no-mild adhesions and moderate-severe adhesions [28].

Statistical analyses were conducted using JMP Pro 14 software (SAS Institute, Cary, NC, USA). Comparisons were made using Fisher's exact test, the Chi-squared test, or the $t$ test, as appropriate. Multivariate logistic regression analyses were conducted to identify the independent risk factors for OO. Odds ratios and $95 \%$ confidence intervals were also estimated. In univariate and multivariate analyses, a value of $p<0.05$ was considered significant.

Appropriate research ethics and review board permissions were obtained from Showa University Northern Yokohama Hospital (Yokohama, Japan; approval number, 19H093). Informed consent was obtained from the patients who were the subjects of this study, and who were given the choice to opt out.

\section{Results}

Table 1 summarizes the patients' clinical characteristics. A history of laparotomy, neoadjuvant therapy, and clinical stage (stage III) were significantly more frequent in the OS group, but other factors did not differ significantly between the OI and OS groups. The use of anti-adhesion material was significantly greater and blood loss was significantly less in the OS group (Table 2). There were no significant differences in operation time, postoperative hospital stay, or period until stoma closure, between the groups (Table 2). The incidence of OO was significantly lower in the OS group (Table 3). There were no significant differences in stomarelated complications, except for bowel obstruction/ileus and $\mathrm{OO}$, or in the degree of intra-abdominal adhesions confirmed during stoma closure, between the groups (Table 3). There were no significant differences in the horizontal or vertical thickness of the rectus abdominis muscle (Fig. 2a, b), or in the length of the straight line connecting both ends of the rectus abdominis muscle, or the position where the stoma limb penetrated the rectus abdominis muscle, in the 64 patients who underwent CT examination before stoma closure (Fig. 2c, d) (Table 3). The angle between the stoma limbs and the rectus abdominis (Fig. 2e) was significantly higher in the OS group (Table 3). Multivariate analysis using logistic regression analysis identified that only the OS position was a significant factor in reducing the incidence of OO (Table 4).
Table 1 The patients' characteristics

\begin{tabular}{llll}
\hline & OI $(n=54)$ & OS $(n=79)$ & $P$ value \\
\hline Sex(M: F) & $41: 13$ & $57: 22$ & 0.6914 \\
Age(years)※ & $63(32-84)$ & $61.0(34-84)$ & 0.6577 \\
Past history of laparotomy & $11(20.4 \%)$ & $21(26.6 \%)$ & 0.0047 \\
BMI $\left(\mathrm{kg} / \mathrm{m}^{2}\right) ※$ & $22.9(15.6-28.8)$ & $22.6(15.4-32.5)$ & 0.7727 \\
Primary disease (RC: NET/UC/FAP) & $45: 9$ & $66: 13$ & 0.9743 \\
Neoadjuvant therapy (done: none) & $6: 48$ & $24: 55$ & 0.0109 \\
Clinical stage (0-II: III) & $41: 13$ & $47: 32$ & 0.0466 \\
PS(0-1: 2-4) & $54: 0$ & $78: 1$ & 0.3062
\end{tabular}

※ median

$R C$ Rectal cancer, NET Neuroendocrine tumor, UC Ulcerative colitis, FAP Familial adenomatous polyposis, BMI Body Mass Index, PS Performance Status 
Table 2 Perioperative comparison between the oral inferior group and the oral superior group

\begin{tabular}{llll}
\hline & OI $(n=54)$ & OS $(n=79)$ & $P$ value \\
\hline Surgical procedure (rectal resection: total colectomy) & $52: 2$ & $75: 4$ & 1.0000 \\
Use of anti-adhesion material (use/not use) & $2: 52$ & $23: 56$ & 0.0002 \\
Operation time(minute)※ & $300.5(185-475)$ & $297.0(162-555)$ & 0.7015 \\
Blood loss(ml)※ & $130(0-946)$ & $60.0(0-752)$ & 0.0122 \\
Postoperative hospital stay(day)※ & $14(7-49)$ & $16(9-130)$ & 0.9633 \\
Period until stoma closure(day)※ & $95(18-569)$ & $93(25-560)$ & 0.8177 \\
\hline
\end{tabular}

※ median

$O I$ oral inferior; $O S$ oral superior
Table 3 Postoperative comparison of outlet obstruction, stomarelated complications, intra-abdominal adhesions, thickness of rectus abdominis muscle thickness (horizontal and vertical), length of the straight line connecting both ends of the rectus abdominis muscle, position where the stoma limb penetrated the rectus abdominis muscle, and the angle formed by the rectus abdominis muscle and stoma limbs, between the oral inferior group and the oral superior group

\begin{tabular}{|c|c|c|c|}
\hline & OI & OS & $P$ value \\
\hline Outlet obstruction & $8 / 54(14.8 \%)$ & $1 / 79(1.3 \%)$ & 0.0032 \\
\hline Stoma-related complications (excluded ileus and outlet obstruction) & $8 / 54(14.8 \%)$ & $11 / 79(13.9 \%)$ & 1.0000 \\
\hline Degree of intra-abdominal adhesions (none/mild: moderate/severe) & 48: 6 & 61: 18 & 0.1092 \\
\hline Rectus abdominis muscle thickness (horizontal) $(\mathrm{mm})(<10 \mathrm{~mm}: \geq 10 \mathrm{~mm})$ & 23: 31 & 34: 45 & 0.9593 \\
\hline Rectus abdominis muscle thickness (vertical) (mm) (<10mm: $\geq 10 \mathrm{~mm})$ & 25: 29 & 42: 37 & 0.4366 \\
\hline Length of the straight line connecting both ends of the rectus abdominis muscle $(\mathrm{mm}) ※$ & $64.5(41.8-95)$ & $64.7(47.7-93.2)$ & 0.9408 \\
\hline $\begin{array}{l}\text { The position where the stoma limb penetrate the rectus abdominis muscle(center-inside: } \\
\text { outer) }\end{array}$ & 21: 5 & $33: 5$ & 0.5187 \\
\hline The angle formed by the rectus abdominis muscle and the stoma $\operatorname{limbs}\left({ }^{\circ}\right) ※$ & 88.1 (49.6-123.9) & $95.95(74.1-124.1)$ & 0.0161 \\
\hline
\end{tabular}

※ median

The position where the stoma limb penetrated the rectus abdominis muscle, and the angle formed by the rectus abdominis muscle and the stoma limbs were measured in the OI group (26 patients) and the OS group (38 patients)

$O I$ oral inferior; $O O$ outlet obstruction; $O S$ oral superior

Table 4 Multivariate analysis conducted using logistic regression analysis revealed that only the oral superior position reduced the incidence of outlet obstruction

\begin{tabular}{lll}
\hline & Odds ratio(95\%CI) & $P$ value \\
\hline Oral superior & $0.1057(0.0055-0.6294)$ & 0.0106 \\
Blood loss & $1.0026(0.9993-1.0060)$ & 0.1069 \\
Past history of laparotomy & $0.3358(0.0165-2.2494)$ & 0.2907 \\
Use of anti-adhesion material & $3.4764 \mathrm{e}-8(0-5.9757)$ & 0.3361 \\
Clinical stage & $0.5907(0.0776-2.9606)$ & 0.5414 \\
\hline
\end{tabular}

$O O$ outlet obstruction

\section{Discussion}

A diverting stoma is constructed to prevent postoperative leakage after rectal cancer surgery [1, 2, 29]. No consensus exists regarding whether ileostomy or colostomy is better, but ileostomy is often selected in Japan [3-7]. In our department, we tend to perform loop ileostomy because it is easy to construct and close. Ileostomy is a common procedure, but surgeons should be aware of stoma-related complications. Examples of stoma-related complications are skin disorders; stoma prolapse, stenosis, or depression; and parastoma hernia. Bowel obstruction is an important complication often associated with ileostomy [4, 8-10, $30,31]$. OO, which is a type of bowel obstruction, shows stricture only in the stoma part. It can delay the resumption of food intake and also the start of adjuvant chemotherapy, and it carries a risk of intestinal injury from the insertion of an intestinal catheter for treatment. Therefore, clinicians may be forced to perform early stoma closure [15, 32]. OO was reported previously as ileus caused by postoperative ileostomy for UC and FAP [11-13]. Anus-preserving surgery and laparoscopic surgery for lower rectal cancer has recently become popular with increasing opportunities to construct a DLI. The number of reports of OO is increasing; however, clinicians are not fully aware of $\mathrm{OO}$ as a complication of DLI [14, 20, 32, 33]. Taking steps to prevent $\mathrm{OO}$ is important when surgeons construct and manage a stoma. Several recent reports $[18,34]$ satisfy conditions 
1 and 2 or 1 and 3, which are defined as follows: (1) symptoms of bowel obstruction/ileus symptoms; (2) relief of bowel obstruction by inserting a decompression catheter trans-stomally; and (3) CT findings of a caliber change in the abdominal wall-penetrating part of the stoma but no other obstruction.

The cause of $\mathrm{OO}$ is still unclear, but bowel obstruction and $\mathrm{OO}$ are more likely to occur with ileostomy than with colostomy [34]. Moreover, intestinal pressure is lower than colonic pressure, which may result in OO, resulting from physical adhesion or relative stenosis at the stoma tunnel by the rectus abdominis muscle [4, 12, 15, 26, 35]. Studies [8-10, 12, 18-20, 27] have also implicated the fascia incision method, mesenteric twist and orientation of the proximal limb, the size of stoma tunnel, and laparoscopic surgery.

As a cause of stenosis related to the rectus abdominis muscle in the stoma tunnel, investigators have mentioned a rectus abdominis muscle thickness of $10 \mathrm{~mm}$ or greater, penetration of the center of the rectus abdominis muscle, a sharp angle between the stoma limbs and the rectus abdominis, and laparoscopic surgery $[26,27]$. These findings are attributed to the fact that the stoma limbs are longer between the rectus abdominal muscles and are easily tightened by the rectus abdominal muscles $[26,27]$. In this study, no significant difference was found between the OI group and the OS group in the thickness and penetration position of the rectus abdominis muscle. Although the angle between the stoma limbs and the rectus abdominis was significantly higher in the OS group, there were only 26 and 38 patients in the OI and OS groups, respectively.

Adhesion of the proximal limb and mesentery to the abdominal wall may be another cause. A study on the fasciotomy method, which compared the vertical incision and the cruciate incision, demonstrated that the cruciate incision results in a larger stoma tunnel [18]. However, OO may occur because of adhesion and bending of the proximal limb at the cruciate incision [18]. We encountered no such cases in this series because all patients had a vertical incision. The use of anti-adhesion agents such as Seprafilm [Baxter International Inc.]) was significantly higher in the OS group, but there was no significant difference between the groups in the degree of intra-abdominal adhesions checked at the time of stoma closure [35].

The size of the stoma tunnel is generally sufficiently large to allow two lateral fingers to pass, although individual differences exist in the size of the two lateral fingers [8-10, 12, 18, 27]. Moreover, muscle relaxation occurs during surgery and as laparoscopic surgery is performed under pneumoperitoneal conditions, these features tend to be risk factors. In the present study, open surgery was performed in both the OI and OS groups and OO rarely occurred; therefore, constructing a sufficient stoma tunnel is important so that the stoma limbs can be passed through with a margin to prevent postoperative stenosis of the stoma tunnel.

When constructing loop ileostomy, the oral side is often constructed at 6 o' clock (OI) to prevent the inflow of stool into the anal side and to facilitate self-care [18, 21, 27]. However, reports indicate that $\mathrm{OO}$ is reduced by constructing the oral side at 3 o'clock to induce mesenteric twist [18, 19], although when we observed the inside of the abdominal cavity after pulling the ileum through to construct a DLI during laparoscopic surgery, the mesenteric twist appeared not to be very strong, despite the direction of the proximal limb. We believe that ensuring the stoma limbs do not bend or have stenosis where they penetrate the abdominal wall is important for the prevention of $\mathrm{OO}$.

We speculate that the low incidence of OO in our OS group can be explained as follows: As the oral side intestine of the DLI falls into the pelvic cavity postoperatively when the patient is in an upright position, the pooling of stool in the oral side of the intestine results in further gravitational pull toward the caudal side of the proximal limb. Thus, in the OI group, the proximal limb tended to bend where it transitions from the stoma tunnel into the abdominal cavity. Conversely, in the OS group, the anal limb is more compressed if the oral side is pulled more in the caudal direction (Fig. 3, schema). Thus, the proximal limb seems to be able to use the size of the stoma tunnel more effectively and is less likely to narrow.

As the rectus abdominis muscle thickens, the angle from the stoma tunnel to the abdominal cavity becomes steeper. We believe this factor is consistent with previously reported risk factors [26, 27].

Few reports mention the orientation of the proximal limb among the research factors, with no significant difference observed, owing to the small number of cases [20, 26]. Moreover, with regard to DLI, no study has examined whether the proximal limb was oriented to the 12 o'clock position (OS) to prevent OO.

A limitation of this study is that the type of stoma (ileostomy or colostomy) and stoma construction method (OI or OS) may differ, depending on the operator. Moreover, the construction may have been affected by individual differences in the amount of food, digestive and absorptive capacity, and activity and there were possible individual differences in the size of the stoma tunnel (approximately two lateral fingers) in each patient. As OO may still have unknown pathologies, additional prospective studies using a large sample size are needed. However, we believe that the oral superior DLI was effective in preventing postoperative $\mathrm{OO}$. 
Fig. 3 Schema of the stoma limbs in the oral superior method and the oral inferior method with the patient in an upright position

\section{Ol method}

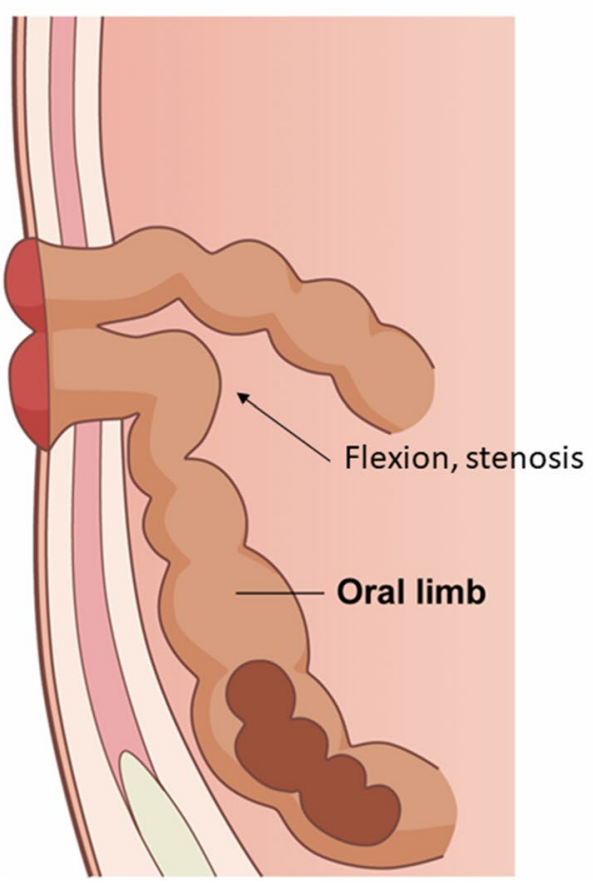

OS method

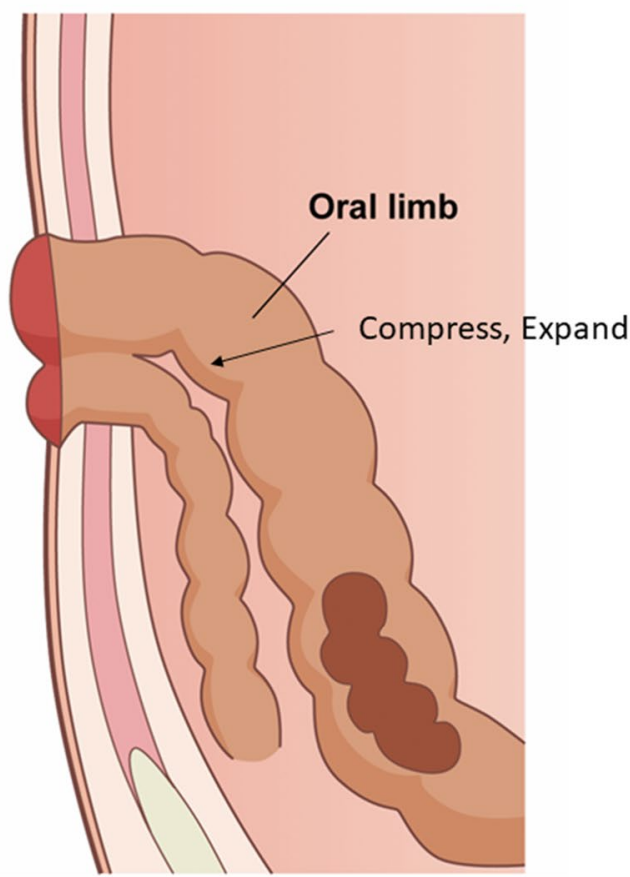

\section{Conclusion}

This study demonstrated that orienting the proximal limb at the OS position (12 o'clock) when constructing a DLI was a significant factor in preventing $\mathrm{OO}$.

\section{Acknowledgements None.}

Funding None.

\section{Declarations}

Conflict of interest We have no conflicts of interest to declare.

Open Access This article is licensed under a Creative Commons Attribution 4.0 International License, which permits use, sharing, adaptation, distribution and reproduction in any medium or format, as long as you give appropriate credit to the original author(s) and the source, provide a link to the Creative Commons licence, and indicate if changes were made. The images or other third party material in this article are included in the article's Creative Commons licence, unless indicated otherwise in a credit line to the material. If material is not included in the article's Creative Commons licence and your intended use is not permitted by statutory regulation or exceeds the permitted use, you will need to obtain permission directly from the copyright holder. To view a copy of this licence, visit http://creativecommons.org/licenses/by/4.0/.

\section{References}

1. Tan WS, Tang CL, Shi L, Eu KW. Meta-analysis of defunctioning stomas in low anterior resection for rectal cancer. Br J Surg. 2009;96:462-72.

2. Matthiessen P, Hallböök O, Rutegård J, Simert G, Sjödahl R. Defunctioning stoma reduces symptomatic anastomotic leakage after low anterior resection of the rectum for cancer: a randomized multicenter trial. Ann Surgery. 2007;246:207-21.

3. Edwards DP, Leppington-Clarke A, Sexton R, Heald RJ, Moran BJ. Stoma-related complications are more frequent after transverse colostomy than loop ileostomy: a prospective randomized clinical trial. Br J Surg. 2001;88:360-3.

4. Law WL, Chu KW, Choi HK. Randomized clinical trial comparing loop ileostomy and loop transverse colostomy for faecal diversion following total mesorectal excision. Br J Surg. 2002;89:704-8.

5. Tilney HS, Sains PS, Lovegrove RE, Reese GE, Heriot AG, Tekkis PP. Comparison of outcomes following ileostomy versus colostomy for defunctioning colorectal anastomoses. World J Surg. 2007;31:1142-51.

6. Rondelli F, Reboldi P, Rulli A, Barberini F, Guerrisi A, Izzo L, et al. Loop ileostomy versus loop colostomy for fecal diversion after colorectal or coloanal anastomosis: a meta-analysis. Int J Colorectal Dis. 2009;24:479-88.

7. Tomita N, Watanabe M, Kameyama M, Takao Y, Sunouchi $\mathrm{K}$, Ogata $\mathrm{Y}$, et al. Questionnaire survey on very low resection with peranal coloanal anastomosis for lower rectal cancer (in Japanese). Nippon Daicho Komonbyo Gakkai Zasshi (J Jpn Soc Colorectal Dis). 2005;58:1-12.

8. Shabbir J, Britton DC. Stoma complications: a literature overview. Colorectal Dis. 2010;12:958-64. 
9. Leong AP, Londono-Schimmer EE, Phillips RK. Life-table analysis of stomal complications following ileostomy. Br J Surg. 1994;81:727-9.

10. Shellito PC. Complications of abdominal stoma surgery. Dis Colon Rectum. 1998;41:1562-72.

11. Ritchie JK. Ulcerative colitis treated by ileostomy and excisional surgery. Fifteen years' experience at St. Mark's Hospital Br J Surg. 1972;59:345-51.

12. $\mathrm{Ng} \mathrm{KH}, \mathrm{Ng}$ DC, Cheung HYS, Wong JCH, Yau KKK, Chung CCC, et al. Obstructive complications of laparoscopically created defunctioning ileostomy. Dis Colon Rectum. 2008;51:1664-8.

13. Francois Y, Dozois RR, Kelly KA, Beart RW Jr, Wolff BG, Pemberton JH, et al. Small intestinal obstruction complicating ileal pouch-anal anastomosis. Ann Surg. 1989;209:46-50.

14. Fujii T, Morita H, Sutoh T, Yajima R, Tsutsumi S, Asao T, et al. Outlet obstruction of temporary loop diverting ileostomy. Hepatogastroenterology. 2015;62:602-5.

15. Okada S, Hata K, Emoto S, Murono K, Kaneko M, Sasaki K, et al. Elevated risk of stoma outlet obstruction following colorectal surgery in patients undergoing ileal pouch-anal anastomosis: a retrospective cohort study. Surg Today. 2018;48:1060-7.

16. Eto K, Kosuge M, Ohkuma M, Noaki R, Neki K, Ito D, et al. Defunctioning ileostomy is a key risk factor for small bowel obstruction after colorectal cancer resection. Anticancer Res. 2018;38:1789-95.

17. Tamura K, Matsuda K, Yokoyama S, Iwamoto H, Mizumoto Y, Murakami D, et al. Defunctioning loop ileostomy for rectal anastomoses: predictors of stoma outlet obstruction. Int J Colorectal Dis. 2019;34:1141-5.

18. Uchino M, Ikeuchi H, Bando T, Matsuoka H, Matsumoto T, Takesue Y, et al. Efficacy of a cross incision at the rectal muscle fascia and ileostomy rotation for prevention of outlet obstruction at the ostomy site in restorative proctocolectomy for ulcerative colitis (in Japanese). Nippon Daicho Komonbyo Gakkai Zasshi (J Jpn Soc Colorectal Dis). 2011;64:73-7.

19. Marcello PW, Roberts PL, Schoetz DJ Jr, Coller JA, Murray JJ, Veidenheimer MC. Obstruction after ileal pouch-anal anastomosis: a preventable complication? Dis Colon Rectum. 1993;36:1105-11.

20. Ohira G, Miyauchi H, Hayano K, Kagaya A, Imanishi S, Tochigi $\mathrm{T}$, et al. Incidence and risk factor of outlet obstruction after construction of ileostomy. J Anus Rectum Colon. 2018;2:25-30.

21. Fasth S, Hultén L. Loop ileostomy: a superior diverting stoma in colorectal surgery. World J Surg. 1984;8:401-7.

22. Schofield PF, Cade D, Lambert M. Dependent proximal loop colostomy: does it defunction the distal colon? Br J Surg. 1980;67:201-2.

23. Winslet MC, Drolc Z, Allan A, Keighley MR. Assessment of the defunctioning efficiency of the loop ileostomy. Dis Colon Rectum. 1991;34:699-703.

24. Dindo D, Demartines N, Clavien PA. Classification of surgical complications: a new proposal with evaluation in a cohort of 6336 patients and results of a survey. Ann Surg. 2004;240:205-13.
25. Brierley JD, Gospodarowicz MK. UICC TNM classification of malignant tumours. Digestive system tumours. 8th ed. John Wiley and Sons Ltd; 2017.

26. Kanazawa A, Shiozawa M, Inagaki D, Sugano N, Akaike M, Imada T. A study on postoperative ileus in patients with ileostomy as a diverting stoma after low anterior resection (in Japanese). Nippon Daicho Komonbyo Gakkai Zasshi (J Jpn Soc Colorectal Dis). 2009;62:497-501.

27. Yoshida Y, Morikawa M, Kurebayashi H, Yokoi S, Koneri K, Murakami M, et al. Risk factors and preventive measures for outlet obstruction after temporary ileostomy construction during rectal resection (in Japanese). J Jpn Surg Assoc. 2018;79:2389-96.

28. Kusunoki M, Ikeuchi H, Yanagi H, Noda M, Tonouchi H, Mohri $\mathrm{Y}$, et al. Bioresorbable hyaluronate-carboxymethylcellulose membrane (Seprafilm) in surgery for rectal carcinoma: a prospective randomized clinical trial. Surg Today. 2005;35:940-5.

29. Hüser N, Michalski CW, Erkan M, Schuster T, Rosenberg R, Kleeff J, et al. Systematic review and meta-analysis of the role of defunctioning stoma in low rectal cancer surgery. Ann Surg. 2008:248:52-60.

30. Arenas Villafranca JJ, López-Rodríguez C, Abilés J, Rivera R, GándaraAdán N, Utrilla NP. Protocol for the detection and nutritional management of high-output stomas. Nutr J. 2015;14:45.

31. Gastinger I, Marusch F, Steinert R, Wolff S, Koeckerling F, Lippert $\mathrm{H}$, et al. Protective defunctioning stoma in low anterior resection for rectal carcinoma. Br J Surg. 2005;92:1137-42.

32. Mizushima T, Kameyama H, Watanabe K, Kurachi K, Fukushima $\mathrm{K}$, Nezu R, et al. Risk factors of small bowel obstruction following total proctocolectomy and ileal pouch anal anastomosis with diverting loop-ileostomy for ulcerative colitis. Ann Gastroenterol Surg. 2017;1:122-8.

33. Fujii T, Tsutsumi S, Matsumoto A, Fukasawa T, Tabe Y, Yajima $\mathrm{R}$, et al. Thickness of subcutaneous fat as a strong risk factor for wound infections in elective colorectal surgery: impact of prediction using preoperative CT. Dig Surg. 2010;27:331-5.

34. Hisamitsu K. Postoperative outlet obstruction in patients with ileostomy (in Japanese). Nippon Daicho Komonbyo Gakkai Zasshi (J Jpn Soc Colorectal Dis). 2018;71:152-6.

35. Nitta T, Ohta M, Kataoka J, Fujii K, Komada K, Ishibashi T. Closure of ileostomy in a patient who underwent loop ileostomy and developed outlet obstruction that improved and then worsened (in Japanese). Nippon Daicho Komonbyo Gakkai Zasshi (J Jpn Soc Colorectal Dis). 2019;72:534-8.

Publisher's Note Springer Nature remains neutral with regard to jurisdictional claims in published maps and institutional affiliations. 